\title{
Prognostic significance of the brachial-ankle pulse wave velocity in patients with essential hypertension: final results of the J-TOPP study
}

\author{
Masanori Munakata, Satoshi Konno, Yukio Miura and Kaoru Yoshinaga, on behalf of the J-TOPP Study \\ Group $^{1}$
}

Brachial-ankle pulse wave velocity (baPWV) is a new tool for measuring arterial stiffness. The prognostic significance of this measure, however, is not fully established. We initiated a multicenter cohort study to examine the prognostic significance of baPWV in patients with essential hypertension in 2002. After baseline measurements were obtained, 662 previously untreated patients (mean age $60 \pm 12$ years, mean blood pressure $156 \pm 19 / 94 \pm 12 \mathrm{~mm} \mathrm{Hg}, 45 \%$ men) underwent long-term follow-up according to the current hypertension treatment guidelines. During the follow-up period (mean: 3 years, range: 3 months8 years), 24 cardiovascular events were observed. The subjects were divided into high and low baPWV groups according to the median value $\left(1750 \mathrm{~cm} \mathrm{~s}^{-1}\right)$. Patients in the high baPWV group were older and had a lower body mass index, higher blood pressure, faster heart rate and higher fasting glucose and plasma creatinine concentrations compared with those in the low baPWV group. Cardiovascular morbidities per 1000 person-years for the high and low baPWV groups were 17.48 and 6.38 , respectively $(P<0.05)$, and the 8-year cardiovascular event-free survival rates were $78.2 \%$ and $93.5 \%$, respectively (log-rank test, $\boldsymbol{P}=\mathbf{0 . 0 1}$ ). A multivariate Cox proportional hazard analysis showed that high baPWV compared with low baPWV was associated with a significantly poorer outcome (hazard ratio (HR) 2.97; $95 \% \mathrm{Cl}: 1.006-9.380$ ). In conclusion, baPWV is an independent risk factor for future cardiovascular events in patients with essential hypertension.

Hypertension Research (2012) 35, 839-842; doi:10.1038/hr.2012.53; published online 26 April 2012

Keywords: arterial stiffness; cardiovascular disease; pulse wave velocity; prognosis

\section{INTRODUCTION}

Hypertension is well known to induce subclinical organ damage in the brain, heart, kidney and blood vessels. This damage can be evaluated using several methods. Among these methods, the measurement of arterial stiffness has recently received a great deal of attention as a marker of vascular damage. ${ }^{1}$ However, carotid-femoral pulse wave velocity (cfPWV) is the 'gold standard' of arterial stiffness measurement, because this parameter has been reported to have prognostic significance in patients with end-stage renal disease $e^{2,3}$ or essential hypertension ${ }^{4}$ and in the general population. ${ }^{5-8}$ Moreover, the latest ESH/ESC hypertension guidelines have shown that a carotid-femoral PWV of $12 \mathrm{~m} \mathrm{~s}^{-1}$ is a marker of organ damage. ${ }^{9}$

Brachial-ankle pulse wave velocity (baPWV) is a new tool for the assessment of arterial stiffness; ${ }^{10,11}$ its implementation is straightforward, and its use has spread rapidly among general practitioners in East Asian countries. ${ }^{12-14}$ However, because this measure is still in its infancy, less evidence on prognostic value is currently available for baPWV than for cfPWV. Recently, we have shown similar areas under the curve for the prediction of stroke or coronary artery disease between cfPWV and baPWV in 2287 Japanese subjects. ${ }^{15}$ Kitahara et al. have shown that high baPWV (that is, $>1960 \mathrm{~cm} \mathrm{~s}^{-1}$ ) is associated with an increased risk of both total and cardiovascular death in end-stage renal disease. ${ }^{16}$ It has also been shown in the general populations that higher baPWV is associated with an increased risk of total death, as well as of cardiovascular death. ${ }^{17,18}$ However, whether increased baPWV is predictive of clinical outcome or death in patients with essential hypertension has not been studied. To address this issue, we performed a prospective, multicenter cohort study titled 'Japanese Trial on the Prognostic Implication of PWV (J-TOPP)' in 2002. ${ }^{19}$ Over an 8-year follow-up period, we found that a higher baPWV is associated with an increased risk of cardiovascular events in this cohort.

\section{METHODS}

Design and study population

Fifty-three institutions agreed to participate in this study (see the Supplementary Information). Patients with essential hypertension, who had never been treated, were eligible for entry into the study when they exhibited 
repeated office blood pressure readings of over $140 / 90 \mathrm{~mm} \mathrm{Hg}$ and were considered in need of antihypertensive treatment. The inclusion and exclusion criteria have been reported previously. ${ }^{19}$ Blood pressure, heart rate and baPWV were measured at baseline after at least $5 \mathrm{~min}$ of supine rest using the AT-form PWV/ABI device (Colin, Komaki, Japan), as has been described elsewhere. ${ }^{11,12}$ After overnight fasting, morning blood was withdrawn from the antecubital vein for the investigation of biochemical variables. Urinary albumin excretion was measured by spot urine sampling.

This study commenced in April 2002; patient recruitment was closed in December 2004. As previously reported, 718 untreated patients with essential hypertension were registered for the J-TOPP study. ${ }^{19}$ After enrollment, the patients were placed on a regimen of antihypertensive medication. They were initially treated with an angiotensin receptor blocker, a calcium channel blocker or an angiotensin-converting enzyme inhibitor for the first 3 months and received additional diuretics or sympatholytic agents to lower their blood pressure to $<140 / 90 \mathrm{~mm} \mathrm{Hg}$. Other classes of drugs were added if their blood pressure was not controlled well under this regime. Other risk factors such as hyperglycemia or dyslipidemia were also treated according to the relevant guidelines.

The primary and secondary end points were urinary albumin excretion and fatal and nonfatal cardiovascular events, respectively. We have already reported that higher baPWV is associated with a higher risk of microalbuminuria 2 years after the initiation of antihypertensive treatment. ${ }^{20}$ In this study, we examined whether higher baPWV is associated with higher cardiovascular morbidity over a longer follow-up period of up to 8 years.

\section{Follow-up survey}

The diagnosis of disease or cause of death was based on hospital record forms or death certificate data, respectively, as reviewed by each author (see Supplementary Information). Information on the cardiovascular events and death was obtained every year by mail. During the follow-up period (mean: 3 years, range: 3 months- 8 years), 24 fatal and nonfatal cardiovascular events were reported, viz. 8 cases of cerebral infarction ( 1 death), 2 cases of cerebral hemorrhage (1 death), 1 case of subarachnoid hemorrhage, 3 cases of myocardial infarction, 9 cases of angina pectoris and 1 case of abdominal aortic aneurysm. The follow-up time was defined from the date of the baseline examination to the date of the first cardiovascular event or death or the date of last contact free of cardiovascular disease.

The investigation conforms to the principles outlined in the Declaration of Helsinki. This study was approved by the medical ethics committee of Tohoku Rosai Hospital, and all patients provided informed consent.

\section{Statistical analysis}

The data were expressed as the mean \pm s.d. or medians with interquartile range for continuous variables and as frequencies (percentages) for categorical measures. The categorical variables included sex, current smoking status and the use of a renin-angiotensin system (RAS) inhibitor. The subjects were divided into two groups (low and high baPWV) according to the median baPWV. The event rates during the follow-up period were expressed as number/1000 patient-years, that is, ratio of the observed number of events to the total number of patient-years of exposure.

Differences in the means were assessed by the Student's $t$-test or the MannWhitney $U$-test; the difference in frequency was examined by $\chi^{2}$ test. The event-free survival rate was compared between the low baPWV and high baPWV groups using the Kaplan-Meier method. The differences in unadjusted survival rates were calculated using the log-rank test. The association between the baseline covariates and the outcomes was assessed using a univariate and multivariate Cox proportional hazards model. Receiver operating characteristic curve analysis was performed to estimate the best baPWV cutoff value for predicting future cardiovascular events. The predictive accuracy was determined based on the area under the curve. The value of maximizing sensitivity minus (1-specificity) was considered as the best cutoff point. Analyses of the data were performed using the JMP software (ver. 9.0, SAS institute, Cary, NC, USA). $P<0.05$ was considered significant.

\section{RESULTS}

Table 1 shows the baseline clinical characteristics of low and high baPWV groups. Patients with high baPWV were statistically significantly older $(P<0.0001)$ and thinner $(P<0.01)$ with higher systolic and diastolic blood pressure $(P<0.0001$ for both), a faster heart rate $(P<0.0001)$, higher fasting glucose levels $(P<0.0001)$ and higher plasma creatinine concentrations $(P<0.01)$ than those of the low baPWV group. The number of events in the low baPWV group was 7 , and the number of events in the high baPWV group was 17; the morbidity rates per 1000 patient-years were 6.38 and 17.48 , respectively $(P<0.05)$.

Figure 1 shows the cardiovascular event-free Kaplan-Meier survival curves for both groups. The 8-year event-free rate was significantly lower in the high baPWV group than in the low baPWV group (logrank test, $P=0.01$ ).

In the univariate Cox proportional hazard model, the HR of the cardiovascular events for the high baPWV group vs. the low baPWV

Table 1 Baseline clinical characteristics of low and high baPWV groups

\begin{tabular}{|c|c|c|c|}
\hline Variable & Low baPWV $(\mathrm{n}=337)$ & High baPWV $(\mathrm{n}=325)$ & $\mathrm{P}$ \\
\hline Range of baPWV & $<1750$ & $1750 \leqq$ & \\
\hline Mean of baPWV $\left(\mathrm{cm} \mathrm{s}^{-1}\right)$ & $1532 \pm 145$ & $2065 \pm 251$ & $<0.0001$ \\
\hline Age (years) & $54.7 \pm 11.0$ & $64.9 \pm 11.4$ & $<0.0001$ \\
\hline Men (\%) & 45.4 & 45.2 & NS \\
\hline BMI $\left(\mathrm{kg} \mathrm{m}^{-2}\right)$ & $24.8 \pm 3.4$ & $24.0 \pm 3.5$ & $<0.01$ \\
\hline Systolic blood pressure (mm Hg) & $149.6 \pm 16.2$ & $163.6 \pm 19.2$ & $<0.0001$ \\
\hline Diastolic blood pressure $(\mathrm{mm} \mathrm{Hg})$ & $91.7 \pm 11.0$ & $95.3 \pm 12.7$ & $<0.0001$ \\
\hline Heart rate (b.p.m.) & $67.3 \pm 10.0$ & $71.0 \pm 11.6$ & $<0.0001$ \\
\hline Total cholesterol $\left(\mathrm{mmol} \mathrm{I}^{-1}\right)$ & $5.43 \pm 0.98$ & $5.40 \pm 0.93$ & NS \\
\hline $\mathrm{LDL}\left(\mathrm{mmol} \mathrm{I}^{-1}\right)$ & $2.51 \pm 1.00$ & $2.44 \pm 1.00$ & NS \\
\hline $\mathrm{HDL}(\mathrm{mmol} \mathrm{I}-1)$ & $1.47 \pm 0.38$ & $1.50 \pm 0.42$ & NS \\
\hline Triglyceride $(\mathrm{mmol} \mathrm{I}-1)$ & $6.22(4.61,9.28)$ & $6.06(4.73,8.90)$ & NS \\
\hline Fasting blood glucose $\left(\mathrm{mmol} \mathrm{I}^{-1}\right)$ & $5.50(5.11,5.95)$ & $5.72(5.22,6.34)$ & $<0.0001$ \\
\hline $\mathrm{Cr}\left(\mu \mathrm{mol} \mathrm{I}^{-1}\right)$ & $62.1 \pm 15.4$ & $65.3 \pm 15.3$ & $<0.01$ \\
\hline Current smoker (\%) & 22.1 & 23.0 & NS \\
\hline RAS inhibitor (\%) & 64.7 & 67.6 & NS \\
\hline
\end{tabular}

Abbreviations: baPWV, brachial-ankle PWV; BMI, body mass index; NS, not significant; RAS, renin-angiotensin system. 


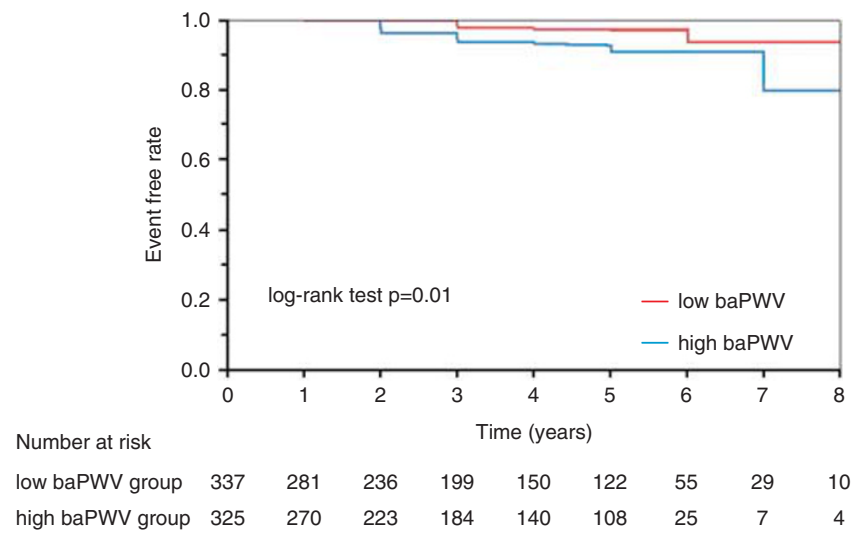

Figure 1 Event-free survival curves for the low and high baPWV groups.

Table 2 Results of univariate Cox regression analysis

\begin{tabular}{|c|c|c|c|}
\hline Variable & $H R$ & $95 \% \mathrm{Cl}$ & $P$ \\
\hline High baPWV (vs. low baPWV) & 2.98 & $1.27-7.81$ & $<0.05$ \\
\hline Age (per 1 year) & 1.04 & $1.01-1.09$ & $<0.01$ \\
\hline Men (vs. Women) & 2.04 & $0.91-4.74$ & NS \\
\hline BMI (per $1 \mathrm{~kg} \mathrm{~m}^{-2}$ ) & 0.93 & $0.82-1.04$ & NS \\
\hline Systolic blood pressure (per 1 mm Hg) & 1.00 & $0.98-1.02$ & NS \\
\hline Diastolic blood pressure (per $1 \mathrm{~mm} \mathrm{Hg}$ ) & 1.00 & $0.96-1.03$ & NS \\
\hline Heart rate (per 1 b.p.m.) & 0.97 & $0.93-1.01$ & NS \\
\hline Total cholesterol (per $1 \mathrm{mmoll}^{-1}$ ) & 0.77 & $0.49-1.19$ & NS \\
\hline LDL (per $1 \mathrm{mmol} \mathrm{I}^{-1}$ ) & 0.98 & $0.91-1.05$ & NS \\
\hline HDL (per $1 \mathrm{mmol}^{-1}$ ) & 0.66 & $0.20-1.83$ & NS \\
\hline Triglyceride (per $1 \mathrm{mmol}^{-1}$ ) & 0.97 & $0.86-1.06$ & NS \\
\hline Fasting blood glucose (per $1 \mathrm{mmol}^{-1}$ ) & 0.78 & $0.45-1.13$ & NS \\
\hline $\operatorname{Cr}\left(\operatorname{per} 1 \mu \mathrm{mol} \mathrm{I}^{-1}\right)$ & 1.02 & $1.00-1.05$ & $<0.05$ \\
\hline Current smoker & 0.89 & $0.25-2.40$ & NS \\
\hline Use of RAS inhibitors & 0.45 & $0.20-0.99$ & $<0.05$ \\
\hline
\end{tabular}

Abbreviations: baPWV, brachial-ankle PWV; BMI, body mass index; HR, hazard ratio; NS, not significant; RAS, renin-angiotensin system.

group was 2.98 (95\% CI: 1.27-7.81). Age (HR 1.04, 95\% CI: 1.012-1.088), plasma creatinine (HR 1.02, 95\% CI: 1.004-1.048) and the use of RAS inhibitors (HR 0.45, 95\% CI: 0.201-1.00) were also significantly associated with cardiovascular prognosis when analyzed using the univariate Cox proportional model (Table 2). In a multivariate Cox proportional model adjusting for age, sex, body mass index, systolic blood pressure, heart rate, fasting blood glucose, total cholesterol, plasma creatinine concentration, smoking status and the use of RAS inhibitors, the HR of the high baPWV group in comparison with the low baPWV group was 2.97 (95\% CI: 1.006-9.380). The absence of RAS inhibitors was also associated with a significantly poorer outcome compared with patients treated with RAS inhibitors (HR 3.58, 95\% CI: 1.44-9.16). ROC analysis has shown that a baPWV of $1763 \mathrm{~cm} \mathrm{~s}^{-1}$ was the best cutoff point for future cardiovascular events (area under the curve 0.620, sensitivity $=70.8 \%$ and specificity $=75.4 \%$; Figure 2 ). The Cox proportional hazards model showed that the group with a baPWV $\geqslant 1763 \mathrm{~cm} \mathrm{~s}^{-1}$ displayed a 3.18 -fold increase in HR compared with the group with a baPWV $<1763 \mathrm{~cm} \mathrm{~s}^{-1}$ after adjustment for age, sex, body mass index, systolic blood pressure, heart rate, fasting blood glucose, total cholesterol, plasma creatinine concentration, smoking status and the use of RAS inhibitors. Thus, an optimal cutoff value

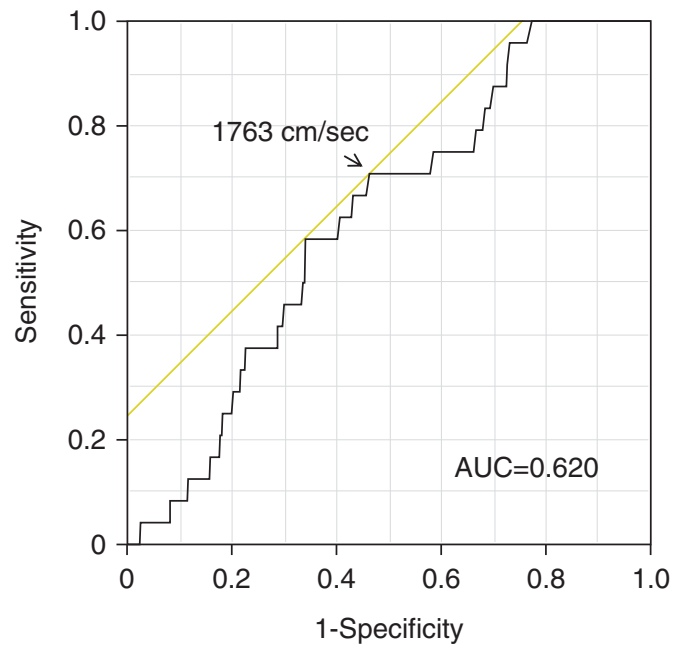

Figure 2 Receiver operating characteristic curve for the onset of cardiovascular events. AUC, area under the curve.

based on ROC analysis slightly increased the point estimate of HR compared with that obtained when the cutoff was determined according to the median value.

\section{DISCUSSION}

The J-TOPP study is the first multicenter cohort study to examine the prognostic significance of baPWV in patients with essential hypertension. When the subjects were dichotomized according to the median value of the total cohort's baPWV $\left(1750 \mathrm{~cm} \mathrm{~s}^{-1}\right)$, the HR in the higher baPWV group was $\sim 3$ times higher compared with that observed in the low baPWV group, even after adjustment for traditional risk factors. The results were similar when we used the best cutoff value according to the ROC analysis: $1763 \mathrm{~cm} \mathrm{~s}^{-1}$ based on the ROC analysis. Thus, baPWV is a novel risk factor for future cardiovascular events in patients with essential hypertension.

The ESH/ESC hypertension guidelines indicated that a cfPWV of $12 \mathrm{~m} \mathrm{~s}^{-1}$ is a marker of organ damage because the cardiovascular prognosis apparently became worse at cfPWV values above $12 \mathrm{~m} \mathrm{~s}^{-1}$. Our study showed that a baPWV of $17.6 \mathrm{~m} \mathrm{~s}^{-1}$ could also be a threshold marker; prognosis becomes worse at higher values. Thus, approximately $17.6 \mathrm{~m} \mathrm{~s}^{-1}$ of baPWV seems to be compatible with a prognostic threshold of $12 \mathrm{~m} \mathrm{~s}^{-1}$ of cfPWV. This hypothesis is further supported by our previous study. In that study of 89 subjects, the correlation between cfPWV and baPWV was good $(r=0.755$, $P<0.00001){ }^{11}$ Regression analysis showed that the baPWV value showed an increase in predictive value of approximately 1.5 times when compared with cfPWV, which suggests that $12 \mathrm{~m} \mathrm{~s}^{-1}$ of cfPWV corresponds to $\sim 18 \mathrm{~m} \mathrm{~s}^{-1}$ of baPWV.

Not only blood pressure but also blood glucose and lipid levels are targeted for strict treatment in the current guidelines for hypertension treatment. Accordingly, neither baseline blood pressure nor baseline blood glucose or lipid levels failed to predict the future cardiovascular events. The baPWV predicted cardiovascular events even in this situation, suggesting that higher baPWV is associated with established, more advanced and less reversible arterial changes.

In this study, the patients were treated according to the current Japanese hypertension treatment guidelines; no specific treatment was given to the high baPWV group. In fact, RAS inhibitors were similarly given to both the low and high baPWV groups. In other words, the 
difference in prognosis observed in both groups cannot be explained by any sort of difference in treatment.

Five previous reports have examined the prognostic significance of baPWV. One studied baPWV in hemodialysis patients, ${ }^{16}$ two were conducted in the general population ${ }^{17,18}$ and the remaining two were conducted in patients with acute coronary syndrome ${ }^{21}$ or diabetes accompanied by coronary artery disease. ${ }^{22}$ Although the cohorts differed extensively, the suggested cut off value for poor cardiovascular prognosis ranged from 1700 to $1963 \mathrm{~cm} \mathrm{~s}^{-1}$. In light of the previous and present results, we propose that a baPWV of $18 \mathrm{~m} \mathrm{~s}^{-1}$ should be considered a tentative marker for organ damage.

It is noteworthy that our study was conducted by multiple centers, representing 53 institutions, whereas the other studies all involved single centers. The number of hospitals and clinics included in this study was compatible (27 vs. 26). Furthermore, the participating institutes covered a broad area extending from Hokkaido to Shikoku. Thus, our cohort includes a broad spectrum of Japanese patients with essential hypertension, independent of geography or scale of the medical institution at which they received treatment, which allows for greater generalization of our results as compared with previous studies.

BaPWV is likely to be influenced by the nature of not only the aorta but also the lower-limb artery. ${ }^{12}$ The prognostic significance of the aortic PWV is well confirmed, but the prognostic value of the femoral-ankle PWV is debatable; ${ }^{23}$ therefore, it has been questioned whether baPWV could, in fact, predict cardiovascular events. We showed in 2287 Japanese subjects that the area under the curve for the prediction of stroke or coronary artery disease was similar between cfPWV and baPWV. ${ }^{15}$ Furthermore, it has been shown in end-stage renal disease and in the general population that baPWV predicts total mortality as well as cardiovascular mortality. ${ }^{16,17}$ Thus, taking those recent reports and our results into consideration, baPWV seems to have prognostic significance similar to that of cfPWV.

Our study has several limitations. First, because the number of events in the current study was small, and the follow-up duration was relatively short, the results of our study should be confirmed by a longer follow-up study with a larger number of participants. Second, all the subjects were Japanese, and thus it is not clear whether the present results are applicable to other ethnic groups. Finally, we failed to clarify how to treat hypertensive patients with high baPWV to improve the prognosis. All these limitations are important and should be addressed in the future.

In conclusion, this is the first multicenter cohort study to demonstrate the prognostic significance of the baPWV in hypertensive patients. The baPWV, a new tool for measuring arterial stiffness, is very easy to implement, and its use has rapidly spread among general practitioners in East Asian countries. We can now conduct a larger-scale, prospective study that includes various ethnic groups; such a study should further confirm the clinical significance of the baPWV measurement.

\section{CONFLICT OF INTEREST}

The authors declare no conflict of interest.

\section{ACKNOWLEDGEMENTS}

This study was supported in part by Grants-in-Aid from the Miyagi Prefectural Kidney Association, the Japan Arteriosclerosis Prevention Fund, and the Japan Labor, Health and Welfare Organization.
1 Laurent S, Boutouyrie P. Arterial stiffness: a new surrogate end point for cardiovascular disease? J Nephrol 2007; 20(Suppl 12): S45-S50.

2 Blacher J, Guerin AP, Pannier B, Marchais SJ, Safar ME, London GM. Impact of aortic stiffness on survival in end stage renal disease. Circulation 1999; 99: 2349-2434.

3 Shoji T, Emoto M, Shinohara K, Kakiya R, Tsujimoto Y, Kishimoto H, Ishimura E, Tabata T, Nishizawa Y. Diabetes mellitus, aortic stiffness, and cardiovascular mortality in end-stage renal disease. J Am Soc Nephrol 2001; 12: 2117-2124.

4 Laurent S, Boutouyrie P, Asmar R, Gautier I, Laloux B, Guize L, Ducimetiere P, Benetos A. Aortic stiffness is an independent predictor of all-cause and cardiovascular mortality in hypertensive patients. Hypertension 2001; 37: 1236-1241.

5 Sutton-Tyrrell K, Najjar SS, Boudreau RM, Venkitachalam L, Kupelian V, Simonsick EM Havlik R, Lakatta EG, Spurgeon H, Kritchevsky S, Pahor M, Bauer D, Newman A Elevated aortic pulse wave velocity, a marker of arterial stiffness, predicts cardiovascular events in well-functioning older adults. Circulation 2005; 111: 3384-3390.

6 Mattace-Raso FU, van der Cammen TJ, Hofman A, van Popele NM, Bos ML, Schalekamp MA, Asmar R, Reneman RS, Hoeks AP, Breteler MM, Witteman JC. Arterial stiffness and risk of coronary heart disease and stroke: the Rotterdam study. Circulation 2006; 113: 657-663.

7 Willum-Hansen T, Staessen JA, Torp-Pedersen C, Rasmussen S, Thijs L, Ibsen H, Jeppesen J. Prognostic value of aortic pulse wave velocity as index of arterial stiffness in the general population. Circulation 2006; 113: 664-670.

8 Mitchell GF, Hwang SJ, Vasan RS, Larson MG, Pencina MJ, Hamburg NM, Vita JA Levy D, Benjamin EJ. Arterial stiffness and cardiovascular events: the Framingham Heart Study. Circulation 2010; 121: 505-511.

9 Mancia G, De Backer G, Dominiczak A, Cifkova R, Fagard R, Germano G, Grassi G, Heagerty AM, Kjeldsen SE, Laurent S, Narkiewicz K, Ruilope L, Rynkiewicz A, Schmieder RE, Boudier HA, Zanchetti A 2007ESH-ESC practice guidelines for the management of arterial hypertension: ESH-ESC task force on the management of arterial hypertension. J Hypertens 2007; 25: 1751-1762.

10 Yamashina A, Tomiyama H, Takeda K, Tsuda H, Arai T, Hirose K, Koji Y, Hori S, Yamamoto $Y$. Validity, reproducibility, and clinical significance of noninvasive brachialankle pulse wave velocity measurement. Hypertens Res 2002; 25: 359-364.

11 Munakata M, Ito N, Nunokawa T, Yoshinaga K. Utility of automated brachial ankle pulse wave velocity measurements in hypertensive patients. Am J Hypertens 2003; 16: 653-657.

12 Munakata M, Nunokawa T, Tayama J, Toyota T. Brachial-ankle pulse wave velocity as a novel measure of arterial stiffness: present evidences and perspectives. Curr Hypertens Rev 2005; 1: 223-234.

13 Seo HS, Kang TS, Park S, Park HY, Ko YG, Choi D, Jang Y, Chung N. Insulin resistance is associated with arterial stiffness in nondiabetic hypertensives independent of metabolic status. Hypertens Res 2005; 28: 945-951.

14 Li B, Gao H, Li X, Liu Y, Wang M. Correlation between brachial-ankle pulse wave velocity and arterial compliance and cardiovascular risk factors in elderly patients with arteriosclerosis. Hypertens Res 2006; 29: 309-314.

15 Tanaka H, Munakata M, Kawano Y, Ohishi M, Shoji T, Sugawara J, Tomiyama H, Yamashina A, Yasuda H, Sawayama T, Ozawa T. Comparison between carotid-femoral and brachial-ankle pulse wave velocity as measures of arterial stiffness. $J$ Hypertens 2009; 27: 2022-2027.

16 Kitahara T, Ono K, Tsuchida A, Kawai H, Shinohara M, Ishii Y, Koyanagi H, Noguchi T, Matsumoto T, Sekihara T, Watanabe Y, Kanai H, Ishida H, Nojima Y. Impact of brachialankle pulse wave velocity and ankle-brachial blood pressure index on mortality in hemodialysis patients. Am J Kidney Dis 2005; 46: 688-696.

17 Miyano I, Nishinaga M, Takata J, Shimizu Y, Okumiya K, Matsubayashi K, Ozawa T, Sugiura T, Yasuda N, Doi Y. Association between brachial-ankle pulse wave velocity and 3-year mortality in community-dwelling older adults. Hypertens Res 2010; 33: $678-682$

18 Turin TC, Kita Y, Rumana N, Takashima N, Kadota A, Matsui K, Sugihara H, Morita Y Nakamura $\mathrm{Y}$, Miura K, Ueshima $\mathrm{H}$. Brachial-ankle pulse wave velocity predicts allcause mortality in the general population: findings from the Takashima study, Japan. Hypertens Res 2010; 33: 922-925.

19 Munakata M, Nunokawa T, Yoshinaga K, Toyota T, J-TOPP Study Group. Brachial-ankle pulse wave velocity is an independent risk factor for microalbuminuria in patients with essential hypertension-a Japanese trial on the prognostic implication of pulse wave velocity (J-TOPP). Hypertens Res 2006; 29: 515-521.

20 Munakata M, Miura Y, Yoshinaga K, J-TOPP study group. Higher brachial-ankle pulse wave velocity as an independent risk factor for future microalbuminuria in patients with essential hypertension: the J-TOPP study. J Hypertens 2009; 27: 1466-1471.

21 Tomiyama H, Koji Y, Yambe M, Shiina K, Motobe K, Yamada J, Shido N, Tanaka N, Chikamori T, Yamashina A. Brachial-ankle pulse wave velocity is a simple and independent predictor of prognosis in patients with acute coronary syndrome. Circ $J$ 2005; 69: 815-822.

22 Nakamura M, Yamashita T, Yajima J, Oikawa Y, Sagara K, Koike A, Kirigaya H, Nagashima K, Sawada H, Aizawa T, Shinken Database Study Group. Brachial-ankle pulse wave velocity as a risk stratification index for the short-term prognosis of type 2 diabetic patients with coronary artery disease. Hypertens Res 2010; 33: 1018-1024.

23 Pannier B, Guérin AP, Marchais SJ, Safar ME, London GM. Stiffness of capacitive and conduit arteries: prognostic significance for end-stage renal disease patients. Hypertension 2005; 45: 592-596. 